\title{
Wideband Bandpass Filter with High Selectivity Based on Dual-Mode DGS Resonator
}

\begin{tabular}{|r|l|}
\hline Journal: & Microwave and Optical Technology Letters \\
\hline Manuscript ID & MOP-16-0283 \\
\hline Wiley - Manuscript type: & Research Article \\
\hline Complete List of Authors: & $\begin{array}{l}\text { Peng, Biao; Beijing University of Posts and Telecommunications, School of } \\
\text { Information and Communication Engineering } \\
\text { Li, Shufang; Beijing University of Posts and Telecommunications, School of } \\
\text { Information and Communication Engineering } \\
\text { Zhu, Jianfeng; Beijing University of Posts and Telecommunications } \\
\text { Zhang, Qianyun; Queen Mary University of London } \\
\text { Deng, Li; Beijing University of Posts and Telecommunications, } \\
\text { Zeng, Qingsheng; University of Ottawa } \\
\text { Gao, Yue; Queen Mary University of London }\end{array}$ \\
\hline Keywords: & $\begin{array}{l}\text { WIDEBAND BANDPASS FILTER, DUAL-MODE DEFECTED GROUND } \\
\text { STRUCTURE RESONATOR, EVEN-/ODD-MODE }\end{array}$ \\
\hline
\end{tabular}




\title{
Wideband Bandpass Filter with High Selectivity Based on Dual-Mode DGS Resonator
}

\author{
Biao Peng, Shufang Li, Jianfeng Zhu, Qianyun Zhang, Li Deng, Qingsheng Zeng and Yue Gao
}

\begin{abstract}
A wideband bandpass filter based on dual-mode defected ground structure resonator (DGSR) is designed and effectively analysed by utilising the even-/odd-mode theory and equivalent circuit models. The lower and upper cutoff frequencies of the filter can be independently tuned by adjusing the evenand odd-mode resonant frequencies of the dual-mode DGSR, respectively. The fabricated prototype is centred at $2.27 \mathrm{GHz}$ with a wide bandwidth (FBW 63.4 \%), low insertion loss (0.386 dB), high selectivity and wide stopband. The attenuation slopes in the lower and upper passband transitions are 108.5 and 136.9 $\mathrm{dB} / \mathrm{GHz}$, respectively. The stopband rejection is better than 22 dB up to $6 \mathrm{GHz}\left(2.6 f_{0}\right)$.
\end{abstract}

Index Terms-Wideband bandpass filter, dual-mode defected ground structure resonator (DGSR), even-/odd-mode.

\section{INTRODUCTION}

W IDEBAND bandpass filters (BPFs) with high quality are important in modern wideband RF systems design. Slot line structures not only show competitive design flexibility owing to the two-dimensional topology, but also have some intrinsic advantages, such as compact size, good selectivity and wide stopband, which make them become increasingly popular recently.

As shown in [1], a fifth-order substrate integrated waveguide BPF with wide bandwidth was realized by cascaded U-shape slots. In [2], two DGSRs were used to design a wideband BPF with excellent selectivity. But the bandwidth of the cascaded structures was limited to tune flexibly. In [3] and [4], based on single DGSR, the two BPFs were designed with wide bandwidth and compact size, however, the passband selectivities of the two filters were not high enough. Based on the even-/odd-mode theory, a novel triple-mode DGSR was proposed to build a wideband BPF with high selectivity in [5], however, the stopband rejection of this filter was relatively low. The novel quad-mode wideband BPF in [6] only had a sharp attenuation slope in the upper passband transition, while the lower passband transition still needs to be improved.

In this letter, a wideband BPF is designed not only with high selectivity but also with flexibly tunable lower and upper cutoff frequencies based on a dual-mode DGSR. A comprehensive equivalent circuit model of the filter is derived to verify the analyses.

Biao Peng, Shufang Li, Jianfeng Zhu and Li Deng are with Beijing University of Posts and Telecommunications, Beijing 100876, China; Corresponding Author: biao.peng@qmul.ac.uk

Biao Peng, Qianyun Zhang and Yue Gao are with Queen Mary University of London, 327 Mile End Road, London E1 4NS, UK

Qingsheng Zeng is with the School of Electrical Engineering and Computer Science (EECS), University of Ottawa, Ottawa, Ontario K1N 6N5, Canada

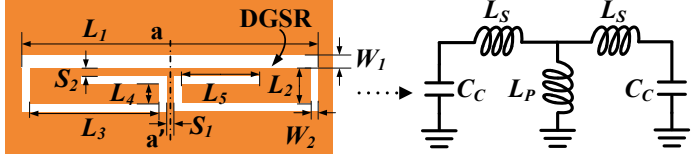

(a)

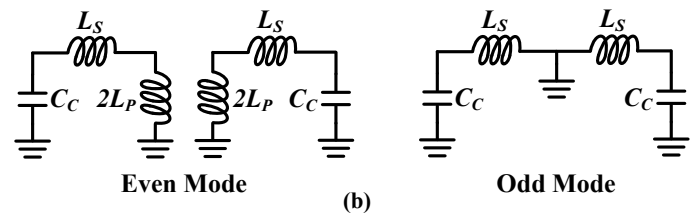

Fig. 1 (a) The layout and equivalent circuit model of the proposed dual-mode DGSR. (b) The equivalent circuit models of the even- and odd-mode. The dimensions are (unit: $\mathrm{mm}$ ): $W_{1}=0.8, W_{2}=0.4, L_{1}=19.6, L_{2}=2.9, L_{3}$ $=8.7, L_{4}=1.7, L_{5}=7.18, S_{1}=0.6$ and $S_{2}=0.8$.

\section{Filter Configuration And Circuit Model}

\section{A. Analyses of the Proposed Dual-Mode DGSR}

Fig. 1(a) (left) depicts the layout of the proposed dualmode DGSR, which is made up of a folded slot line resonator (FSLR) and a coplanar stepped impedance resonator (CSIR). The CSIR is connected to the ground by a narrow metallic strip. The equivalent circuit model of the DGSR is given in Fig. 1(a) (right), where $C_{C}$ models the capacitance between the inner metallic plate and the ground, whose value mainly depends on the circumference of the inner plate and the distance to the ground. $L_{S}$ and $L_{P}$ are the inductances connecting the inner metallic plate to the central inductive path and to the ground, respectively, whose values are directly determined by the width and length of the metallic strips.

Since there is a symmetric plane $\left(a-a^{\prime}\right)$ in the DGSR, the equivalent circuit models of the two modes can be obtained based on even-/odd-mode theory, which are illustrated in Fig. 1(b). The inductance $L_{P}$ has no effect on the odd-mode operation because plane $a-a^{\prime}$ is a virtual ground under oddmode operation. Then, the two resonant frequencies $\left(f_{\text {even }}\right.$, $f_{\text {odd }}$ ) can be calculated as:

$$
\begin{gathered}
f_{\text {even }}=\frac{1}{2 \pi \sqrt{\left(L_{S}+2 L_{P}\right) C_{C}}} \\
f_{\text {odd }}=\frac{1}{2 \pi \sqrt{L_{S} C_{C}}}
\end{gathered}
$$

To clearly demonstrate the high tunability of the two resonant modes, transmission responses of the DGSR are analysed under weakly coupling condition on $0.762 \mathrm{~mm}$-thick Rogers RO4350B with $\epsilon_{r}=3.48$ and $\tan \delta=0.004$ based on the EM-simulator ADS. As Shown in Fig. 2(a), $f_{\text {even }}$ can be independently tuned by adjusting $S_{1}$ because $L_{P}$ changes significantly with the variation of $S_{1}$ while other parameters 


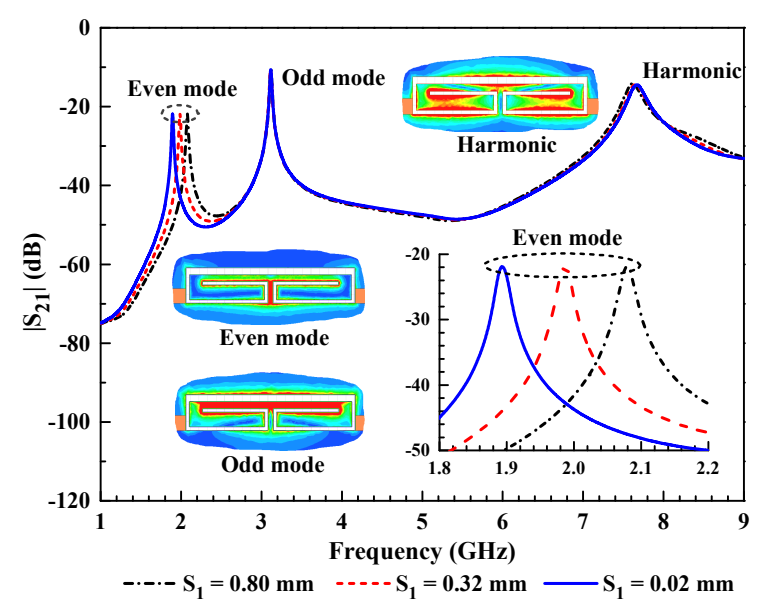

(a)

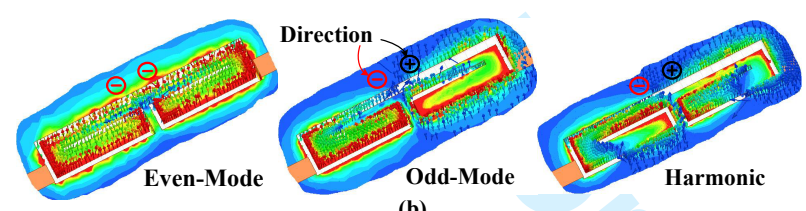

(b)

Fig. 2 (a) $S_{21}$ of the proposed dual-mode DGSR under weakly coupling condition with different $S_{1}$ as parameter, and the current distributions at the three resonant frequencies. (b) Electric fields at the three resonant frequencies (based on the EM-simulator HFSS).

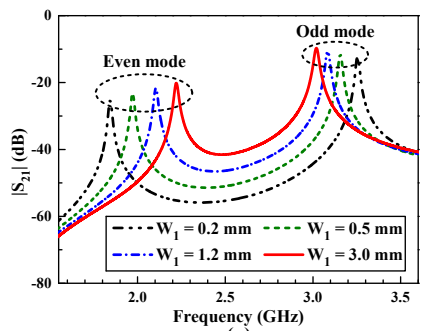

(a)

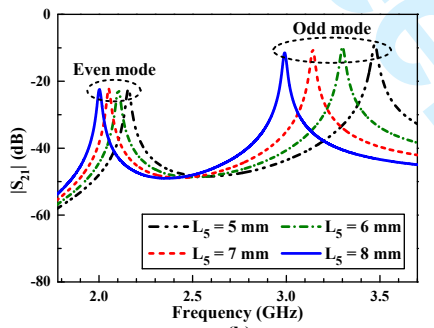

(b)
Fig. 3 (a) $S_{21}$ of the dual-mode DGSR under weakly coupling condition with different $W_{1}$ as parameter. (b) $S_{21}$ of the dual-mode DGSR under weakly coupling condition with different $L_{5}$ as parameter.

nearly remain unchanged. This can also be verified from the current distributions shown in Fig. 2(a), because there is no current flowing through $L_{P}$ under the odd-mode operation. Fig. 2(a) indicates that a harmonic resonance arises at about 7.7 GHz, which is the $1^{\text {st }}$ harmonic of the CSIR. To give a more physical insight, the electric fields at the three resonant frequencies are given in Fig. 2(b). It is clear that the electric fields of the even- and odd-mode along the two sides of $a-a^{\prime}$ are in different directions.

Fig. 3 illustrates that the two modes can be simultaneously tuned by adjusting $W_{1}$ or $L_{5}$, respectively. When $W_{1}$ decreases, decrease of $L_{S}$ is more significant than the increase of $C_{C}$. As a result, the even- and odd-mode resonances move toward lower and higher frequencies, respectively. When $L_{5}$ decreases, the value of $L_{S}$ decreases distinctly, which makes both the even- and odd-mode resonances move towards higher frequencies with the odd-mode moving much faster than the even-mode.
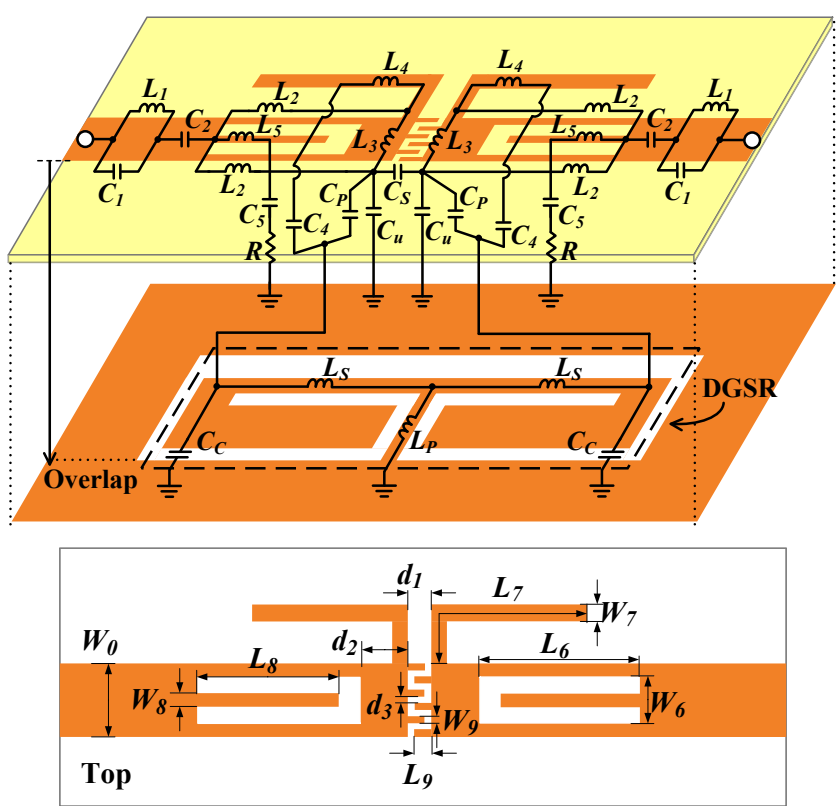

Fig. 4 Topology and equivalent circuit model of the proposed BPF. The dimensions are (unit: $\mathrm{mm}$ ): $W_{0}=1.7, W_{6}=1.1, W_{7}=0.6, W_{8}=0.3$, $W_{9}=0.15, L_{6}=8.4, L_{7}=7.1, L_{8}=8.0, L_{9}=0.45, d_{1}=0.6, d_{2}=2.7$ and $d_{3}=0.15$. The electrical parameters are: $L_{1}=0.69 \mathrm{nH}, L_{2}=0.5 \mathrm{nH}$, $L_{3}=0.1 \mathrm{nH}, L_{4}=1.0 \mathrm{nH}, L_{5}=1.36 \mathrm{nH}, L_{S}=1.8 \mathrm{nH}, L_{P}=1.65 \mathrm{nH}$, $C_{1}=2.08 \mathrm{pF}, C_{2}=6.0 \mathrm{pF}, C_{4}=0.4 \mathrm{pF}, C_{5}=0.53 \mathrm{pF}, C_{S}=0.51 \mathrm{pF}, C_{u}$ $=0.19 \mathrm{pF}, C_{P}=1.5 \mathrm{pF}, C_{C}=0.6 \mathrm{pF}$ and $R=6 \Omega$ (The lumped-element values are obtained based on the method presented in [3]).

\section{B. Design of the Dual-Mode Wideband BPF}

In this section, a wideband BPF is designed based on the proposed dual-mode DGSR. The topology and equivalent circuit model of the proposed BPF are illustrated in Fig. 4. On the top side, $L_{1} / C_{1} / C_{2}$ relate to the input/output ports [4]. To improve the passband selectivity, the sourceload coupling is enhanced by using inter-digital microstrip lines (IDMLs), modelled by $C_{S} / C_{u} / C_{P}$ [2]. $L_{4} / C_{4}$ represent the two open-circuited microstrip stubs, which are bended to realise a broadside coupling from the microstrip feeding lines to the DGSR [7]. From the analyses above, the harmonic resonance would seriously deteriorate the stopband rejection. So two quarter-wavelength $(\lambda / 4)$ embedded open-circuited stubs (EOCSs) are introduced to produce a notch-band around $6 \mathrm{GHz}$ to improve the stopband performance, and they are modelled by series resonators $L_{5} / C_{5} / R$. The EOCSs do not require additional $\mathrm{PCB}$ space while having little effect on the passband performance since the major part of the electric current flows around the edges of microstrip lines [8].

The lower and upper cutoff frequencies of the proposed BPF can be flexibly tuned by adjusting the even- and oddmode resonances, respectively. As shown in Fig. 5a, the lower cutoff frequency is tuned by changing $S_{1}$ while the oddmode resonance and upper cutoff frequency keep invariant. As shown in Fig. 5b, to adjust the upper cutoff frequency, the odd-mode resonance is tuned by adjusting $L_{5}$ and $W_{1}$ simultaneously while the lower cutoff frequency is not affected. The two transmission zeros beside the passband $\left(T_{Z 1}\right.$, $T_{Z 2}$ ) are produced due to the source-load coupling. These zeros appear when $Y_{\text {ine }}=Y_{\text {ino }}$ [9] $\left(Y_{\text {ine }}\right.$ and $Y_{\text {ino }}$ are the admittances looking into the feeding port under even- and odd- 


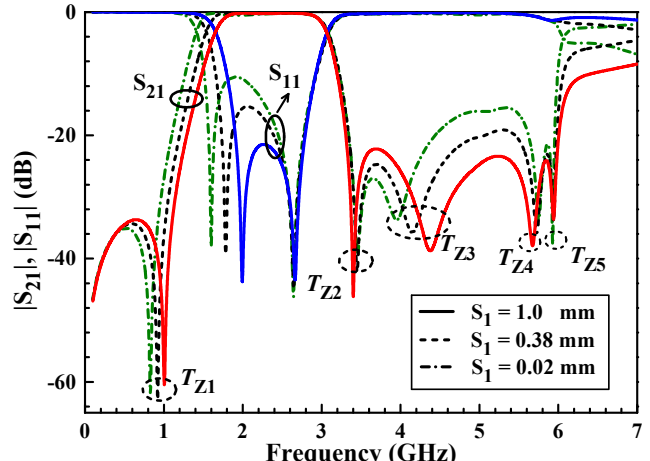

(a)

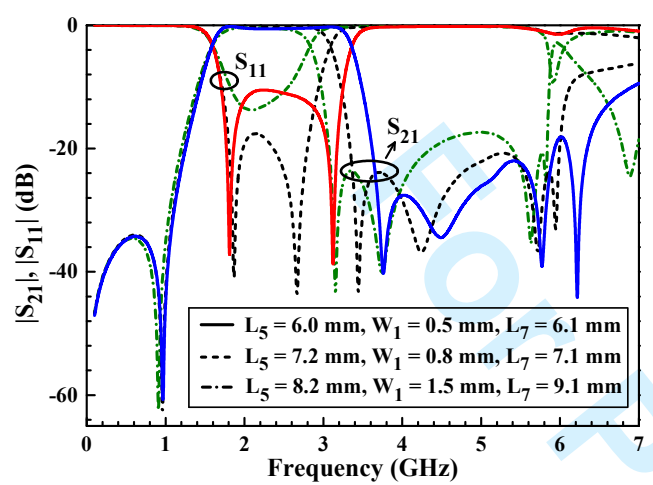

(b)

Fig. 5 (a) Control of the lower cutoff frequencies of the proposed BPF. (b) Control of the upper cutoff frequencies of the proposed BPF.

mode operation, respectively), and can be effectively tuned by adjusting IDMLs. The two $\lambda / 4$ EOCSs produce another two transmission zeros around $6 \mathrm{GHz}\left(T_{Z 4}, T_{Z 5}\right)$, which can be easily tuned by adjusting the length of the EOCSs. This topology also introduces a transmission zero around $4.2 \mathrm{GHz}$ $\left(T_{Z 3}\right)$, which is important to enhance the stopband rejection.

\section{EXPERIMENTAL RESUlTS AND ANALYSES}

A fabricated BPF is measured using Agilent N5244A network analyser. EM-/LC-simulated, measured S-parameters and the group delay of the filter are plotted in Fig. 6(b), which shows a good agreement between measurements and simulations. The bandwidth discrepancies less than $3 \%$ are mainly attributed to the manufacturing errors and the dielectric constant. The measured centre frequency $\left(f_{0}\right)$ is $2.27 \mathrm{GHz}$ with a 3-dB fractional bandwidth (FBW) of $63.4 \%$. The typical insertion loss (IL) is $0.386 \mathrm{~dB}$, and the return loss (RL) is better than $15 \mathrm{~dB}$ within the passband. Lower and upper passband attenuation slopes are 108.5 and $136.9 \mathrm{~dB} / \mathrm{GHz}$, respectively. The group delay at $f_{0}$ is less than $0.5 \mathrm{~ns}$ and its variation within the passband is less than $0.5 \mathrm{~ns}$. Compared with other filters listed in Table I, the proposed dual-mode wideband BPF exhibits competitive advantages. Furthermore, it shows a good stopband up to $6.0 \mathrm{GHz}\left(2.6 f_{0}\right)$ with rejection better than $22 \mathrm{~dB}$.

\section{CONCLUSION}

This letter presented the design and analyses of a wideband BPF based on a dual-mode DGSR, which has been effectively
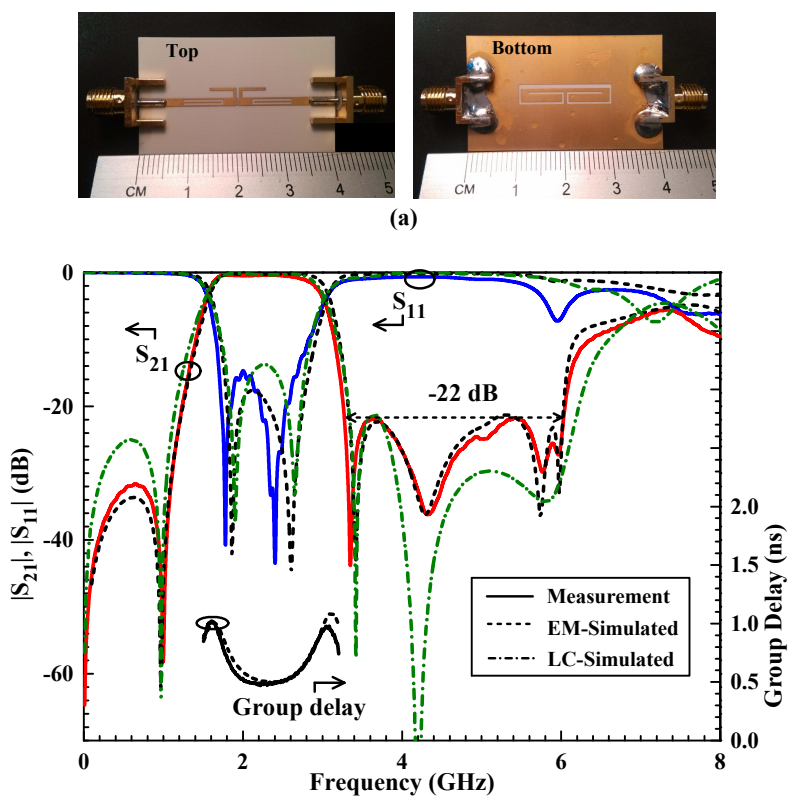

(b)

Fig. 6 (a) Photographs of the fabricated BPF. (b) S-parameters achieved with EM-simulated, LC-simulated and measurement and the group delay of the proposed dual-mode wideband BPF.

TABLE I Comparison Between Relative Filters.

\begin{tabular}{c|c|c|c|c|c}
\hline $\begin{array}{c}\text { Ref. } \\
\text { No. }\end{array}$ & $\begin{array}{c}f_{0} \\
(\mathrm{GHz})\end{array}$ & $\begin{array}{c}\text { FBW } \\
(\%)\end{array}$ & $\begin{array}{c}\text { IL@ } f_{0} \\
(\mathrm{~dB})\end{array}$ & $\begin{array}{c}\text { Lower skirt } \\
(\mathrm{dB} / \mathrm{GHz})\end{array}$ & $\begin{array}{c}\text { Upper skirt } \\
(\mathrm{dB} / \mathrm{GHz})\end{array}$ \\
\hline$[1]$ & 8.5 & 42 & 1.1 & 25 & 19 \\
{$[2]$} & 5.0 & 75 & 0.4 & 262.2 & 75.9 \\
{$[3]$} & 2.23 & 62 & 0.27 & 59.8 & 49.6 \\
{$[4]$} & 2.15 & 84 & 2.3 & 76.1 & 77.0 \\
{$[5]$} & 3.5 & 16 & 1.9 & 180 & 86 \\
{$[6]$} & 2.45 & 32 & 1.98 & 53.1 & 302.0 \\
{$[7]$} & 2.5 & 60 & 0.9 & 53.4 & 37.0 \\
This & $\mathbf{2 . 2 7}$ & $\mathbf{6 3 . 4}$ & $\mathbf{0 . 3 8 6}$ & $\mathbf{1 0 8 . 5}$ & $\mathbf{1 3 6 . 9}$ \\
work & & & & & \\
\hline
\end{tabular}

* Lower/upper skirt is calculated as $\left|\alpha_{\max }\right|-\left|\alpha_{\min }\right| /\left|f_{z}-f_{c}\right|$, where $\alpha_{\max }$ is the attenuation of the first out-of-band transmission zero and $\alpha_{\min }$ is the 3 $\mathrm{dB}$ attenuation; $f_{z}$ is the frequency of the first out-of-band transmission zero and $f_{c}$ is the $3 \mathrm{~dB}$ cutoff frequency. Lower/upper skirt for reported ones is estimated from the figures in the papers.

analysed using the even-/odd-mode theory and equivalent circuit models. The even- and odd-mode resonances of the DGSR can be easily tuned, leading to the flexibly tunable lower and upper cutoff frequencies of the proposed BPF. The fabricated filter has shown competitive performance such as high selectivity, low insertion loss and wide stopband.

\section{ACKNOWLEDGEMENT}

This work was supported by the major special instrument projects of National Natural Science Foundation of China (NSFC) under Grant [61427801]

\section{REFERENCES}

[1] R. S. Chen, S.-W. Wong, L. Zhu, and Q.-X. Chu, "Wideband bandpass filter using u-slotted substrate integrated waveguide (siw) cavities," IEEE Microw. Wireless Compon. Lett., vol. 25, no. 1, pp. 1-3, Jan. 2015. 
[2] X. Luo, H. Qian, J.-G. Ma, and E.-P. Li, "Wideband bandpass filter with excellent selectivity using new csrr-based resonator," Electron. Lett., vol. 46, no. 20, pp. 1390-1391, Sep. 2010.

[3] A. Ebrahimi, W. Withayachumnankul, S. Al-Sarawi, and D. Abbott, "Compact dual-mode wideband filter based on complementary split-ring resonator," IEEE Microw. Wireless Compon. Lett., vol. 24, no. 3, pp. 152154, March 2014.

[4] J.-C. Liu, H.-C. Lin, B.-H. Zeng, K.-D. Yeh, and D.-C. Chang, "An improved equivalent circuit model for csrr-based bandpass filter design with even and odd modes," IEEE Microw. Wireless Compon. Lett., vol. 20, no. 4, pp. 193-195, Apr. 2010.

[5] H. Liu, L. Shen, Y. Jiang, X. Guan, S. Wang, L. Shi, and D. Ahn, "Triplemode bandpass filter using defected ground waveguide," Electron. Lett., vol. 47, no. 6, pp. 388-389, Mar. 2011.

[6] B. Peng, S. Li, J. Zhu, L. Deng, Q. Zeng, and Y. Gao, "Compact quadmode bandpass filter based on quad-mode dgs resonator," IEEE Microw. Wireless Compon. Lett., (Accepted 2016).

[7] M. Mandal and S. Sanyal, "Compact wide-band bandpass filter using microstrip to slotline broadside-coupling," IEEE Microw. Wireless Compon. Lett., vol. 17, no. 9, pp. 640-642, Sep. 2007.

[8] B. Peng, S. Li, B. Zhang, and S. Wang, "Triband filter with high design flexibility and wide stopband using dgs and shorted stub-loaded resonator," Microw. Opt. Tech. Lett., vol. 57, no. 5, pp. 1226-1228, 2015.

[9] J. S. Hong and M. J. Lancaster, "Microstrip filters for rf/microwave applications," New York: Wiley, 2001. 